\title{
Relecture de l'inscription K. 1312 de Prasat Khna, province de Preah Vihear
}

\section{Dominique Soutif et Julia Estève}

\section{OpenEdition}

Journals

Édition électronique

URL : https://journals.openedition.org/baefe/3759

DOI : $10.4000 /$ baefe.3759

ISSN : 2732-687X

Éditeur

ResEFE

Référence électronique

Dominique Soutif, Julia Estève, « Relecture de l'inscription K. 1312 de Prasat Khna, province de Preah Vihear » [notice archéologique], Bulletin archéologique des Écoles françaises à l'étranger [En ligne], Asie du Sud-Est continentale, mis en ligne le 30 août 2021, consulté le 01 septembre 2021. URL : http:// journals.openedition.org/baefe/3759 ; DOI : https://doi.org/10.4000/baefe.3759

Ce document a été généré automatiquement le 1 septembre 2021.

\section{c) $(6)$}

Le Bulletin archéologique des Écoles françaises à l'étranger est mise à disposition selon les termes de la Licence Creative Commons Attribution - Pas d'Utilisation Commerciale - Pas de Modification 4.0 International. 


\title{
Relecture de l'inscription K. 1312 de Prasat Khna, province de Preah
} Vihear

\author{
Dominique Soutif et Julia Estève
}

\section{NOTE DE L'AUTEUR}

Partenariats institutionnels : MESRI, ERC Dharma (European Research Council ;

European Union's Horizon 2020 research and innovation programme, grant agreement $n^{\circ}$ 809994).

Établissement éditeur : EFEO

Établissements porteurs de l'opération : EFEO

Données scientifiques produites:

Dharma Hypothèses

ERC Dharma

1 L'inscription K. 1312 a été signalée pour la première fois dans un mémoire de l'Université Royale des Beaux-Arts ${ }^{1}$ consacré au Prasat Khna, temple situé dans la province de Preah Vihear à une vingtaine de kilomètres au nord/nord-ouest de Tbaeng Meancheay, dans le village de Krala Peas, commune de Preng Tum, district de Choam Ksant $^{2}$ (fig. 1). 
Fig. 1. Localisation du Prasat Khna.

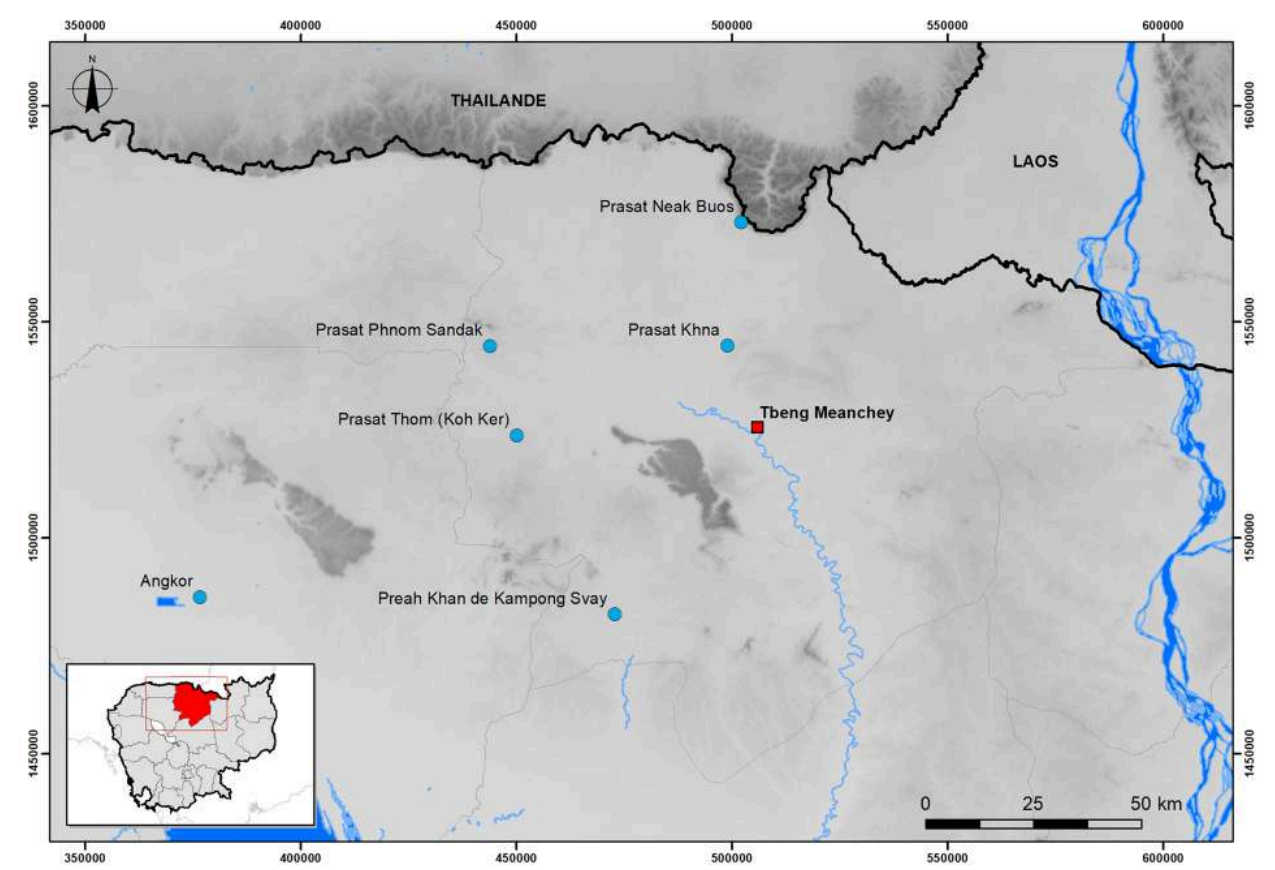

Cartographie : Chea Socheat.

Il s'agit d'un temple important à plus d'un titre. Du fait de sa taille en premier lieu : il comporte en effet trois enceintes couvrant une surface totale de $12000 \mathrm{~m}^{2}$ (300 m nord/ sud $\times 400 \mathrm{~m}$ est/ouest). Les deux premières enceintes accueillent de nombreux bâtiments et l'ensemble présente un plan qualifié de «complexe» par Henri Parmentier (1939, p. 194) qui précisait : « [il] est tellement encombré par la ruine que ses dispositions primitives ne peuvent plus être reconnues sûrement » (fig. $\mathbf{2}^{3}$ ). Dans la première enceinte, un bâtiment rectangulaire et treize sanctuaires de formes variées, auxquels il faut ajouter un gopura oriental en brique à trois accès, ont été identifiés. L'absence de vestiges permet de penser qu'aucun bâtiment - en tout cas pérenne n'était implanté dans la seconde, mais elle comprenait un gopura monumental en grès en avant duquel se trouvait un édicule à stèle. Enfin, la troisième enceinte comportait deux bâtiments que nous proposons, en raison de leur plan singulier, d'identifier aux monuments pérennes caractéristiques des āśrama fondés par Yaśovarman I ${ }^{\text {er }}$ (fin du IX $\mathrm{s}$. de notre ère), les Yaśodharāśrama, et Sūryavarman $\mathrm{I}^{\mathrm{er}}$ (début du $\mathrm{XI}^{\mathrm{e}} \mathrm{s}$. de notre ère), les Vīrāśrama4 . Il s'agit respectivement du bâtiment 'N', à l'est, et du " palais » 'O' situé au nord de la seconde enceinte qui n'avait pas été inventorié par Lunet de Lajonquière. Il faut ajouter à cela un édicule à stèle, le bâtiment 'P', mis au jour en 2020 dans le cadre des travaux du programme de recherche YAŚODHARĀŚRAMA au nord-ouest du bâtiment ' $N$ '. Cette découverte vient soutenir notre identification du bâtiment ' $N$ '. En effet, l'association d'édicules à stèles ${ }^{5}$ à des bâtiments longs de même plan que 'N' est une caractéristique commune des āśrama d'Angkor'. 
Fig. 2. Prasat Khna, plan des deux premières enceintes et localisation des inscriptions.

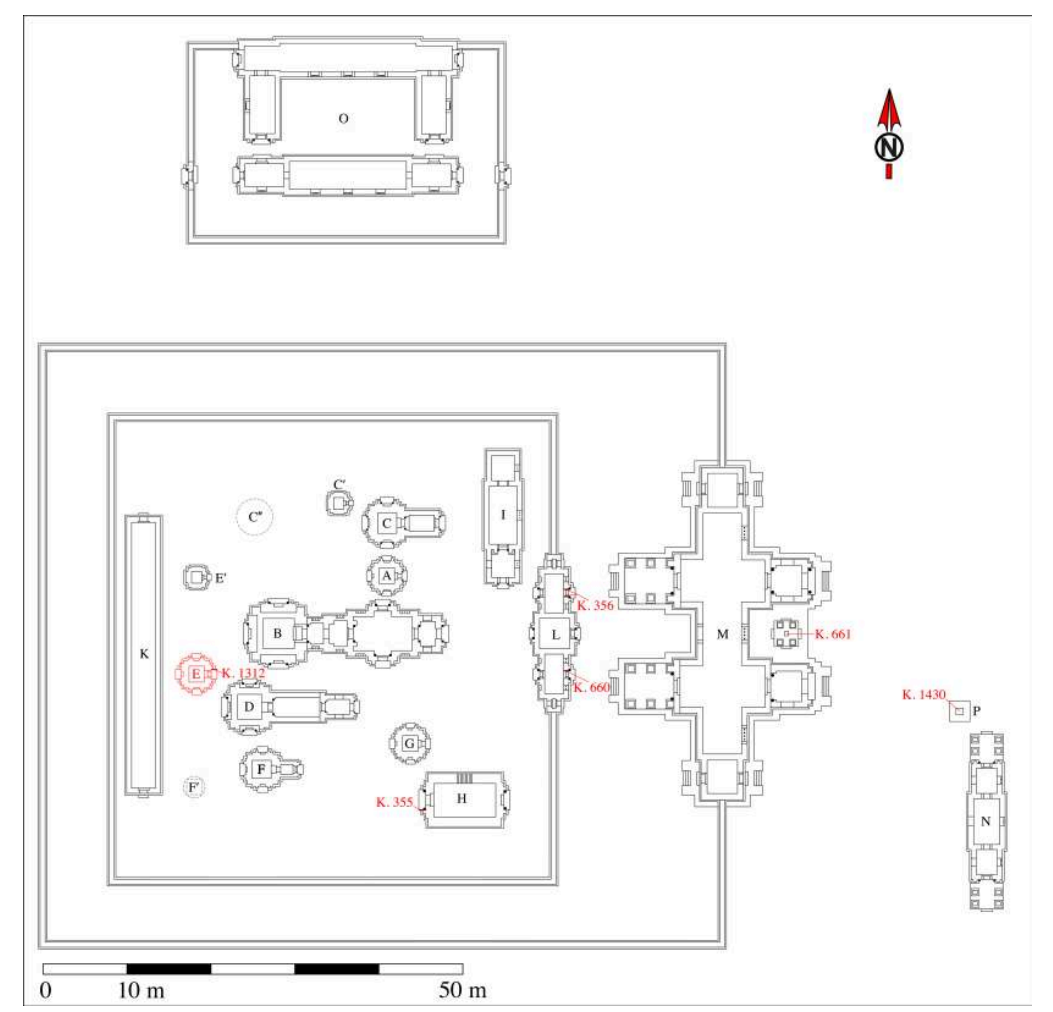

D'après parmentier 1939, pl. XXVII ; BRUGUIER, LACROIX 2013, p. 135, plan 19 et soutiF et al. 2020.

3 Le fait que ces rois aient choisi d'implanter des monastères dans ce temple n'est pas anodin, car ce type de fondations royales était associé à des sites importants, tant pour témoigner de la prodigalité et de la dévotion du roi que de son emprise sur l'ensemble des provinces de l'empire. Son importance ne se dément d'ailleurs pas par la suite puisque Jayavarman VII fonda également l'un de ses hôpitaux à proximité, dont la chapelle, le Prasat Chaeng Meng ${ }^{7}$. Ce vaste ensemble architectural est complété par la présence de plusieurs sanctuaires périphériques, de bassins, et surtout par un baray implanté au nord-est et mesurant $900 \times 250 \mathrm{~m}^{8}$.

D'un point de vue épigraphique, pas moins de sept inscriptions y ont été découvertes à ce jour'. Il s'agit d'un corpus particulièrement important pour un temple provincial. Entre outre, il a connu une certaine notoriété en raison de la mention d'un pustakāśrama, un « āśrama des manuscrits » dans l'inscription K. 355 (bâtiment 'R', porte ouest, piédroit sud, st. XXII ; IX ${ }^{\mathrm{e}}$ s. śaka). Cette occurrence est en effet à l'origine de l'usage de l'appellation pour le moins discutable de «bibliothèque » pour désigner une majorité des sanctuaires annexes des temples angkoriens ${ }^{10}$.

Enfin, le Prasat Khna est également célèbre pour avoir accueilli une trimūrti particulièrement importante composée des kamrateñ 'añ ta 'cas (« l'ancien», Brahmā), śakabrāhmaṇa sakabrāhmaṇa sak brāhma (le "brāhmane śaka», ou "brāhmane scythe », Vișnu) et surtout du kamraten 'añ ta räjya (le Śiva protecteur du royaume). Cette triade est en effet mentionnée dans une ordonnance royale datée de 902 śaka précisant les redevances des "chapelles » (kuți) de Janapada et de Trivikramapada destinées à ces divinités ${ }^{11}$. 
Comme le signalait George Cœdès, le composé janapada est mentionné dans la stèle de Sdok Kak Thom, dans laquelle il est associé au brāhmane Hiranyadāma qui instaura le célèbre culte du Devarāja - ou Kamrateñ Jagat ta Rāja - au sommet du mont Mahendra sous le règne de Jayavarman II. Cœdès notait par ailleurs qu'il peut être compris comme un toponyme, mais aussi comme désignant le "pays d'origine » de ce personnage) ${ }^{12}$. Éric Bourdonneau (2016, p. 123-127) avance que janapada ne désignait pas à l'origine le site de Prasat Khna, mais l'Inde même, d'où serait venu le brāhmane Hiranyadāma, avant de s'installer au Prasat Khna, ce qui explique que ce lieu ait été a posteriori considéré comme le berceau du fondateur de ce culte. L'installation d'un Viṣnu nommé Sakabrāhmaña dans ce temple s'explique alors par le fait qu'il s'agissait de rendre hommage à ce «brāhmane scythe » fondateur du culte du Devarāja, aux côtés de Śiva "protecteur de la royauté $»^{13}$. On notera d'ailleurs que, des sept inscriptions qui mentionnent le śakabrāhmaṇa, trois proviennent de Prasat Khna, et qu'il était le bénéficiaire d'abondantes donations, ce qui souligne encore son prestige ${ }^{14}$.

7 L'inscription K. 1312 est gravée sur le piédroit nord de la tour en brique 'E', qui est ouverte à l'est. Elle est située derrière la tour principale 'B' mais n'est pas dans son alignement : elle est nettement décalée vers le sud (fig. 3).

Fig. 3. Modélisation 3D vestiges des tours $B, D, E, F$ et du mur occidental du bâtiment $K$ de Prasat Khna, vues du nord-est.

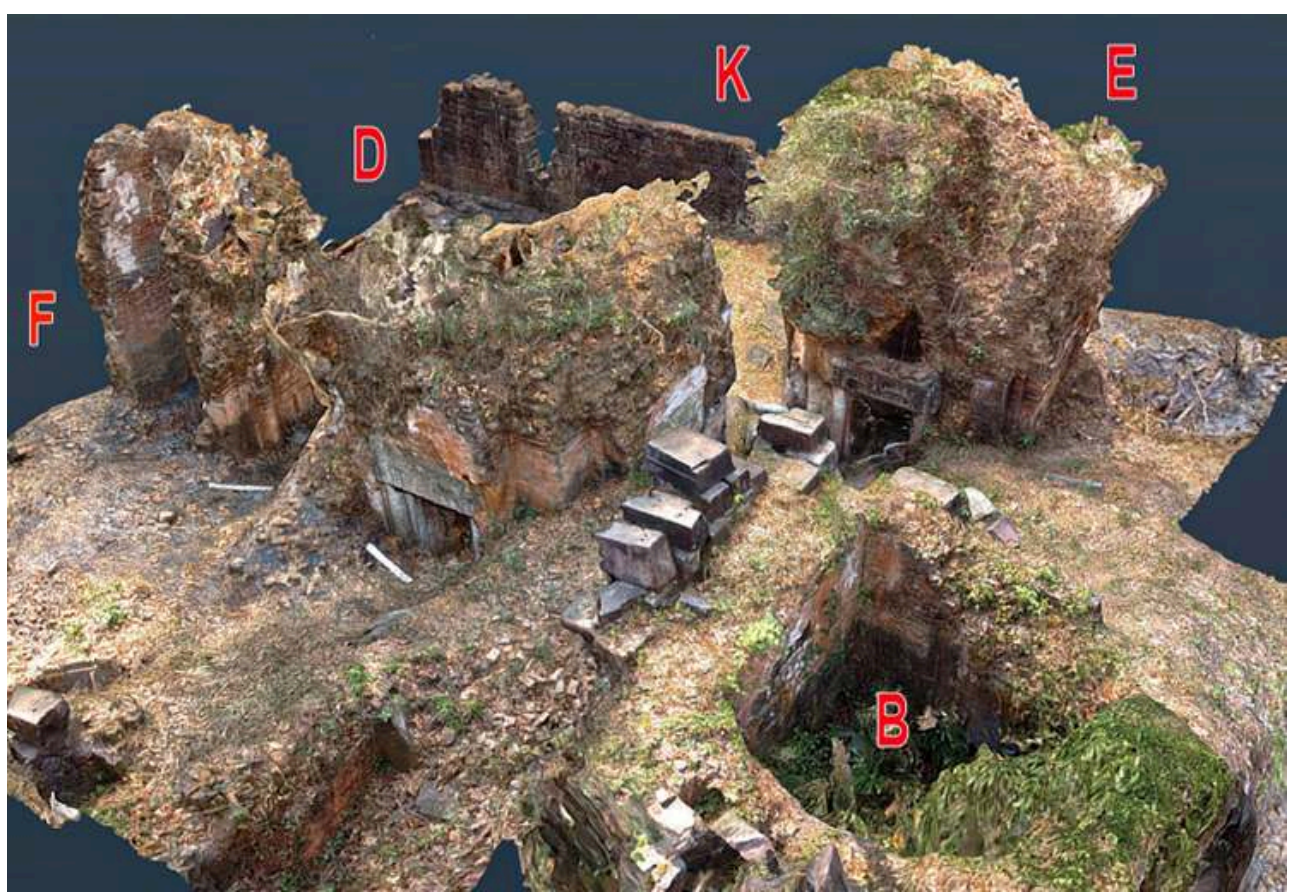

Clichés et modélisation : Nicolas Josso.

Curieusement, bien que cette épigraphe soit sans doute la mieux conservée du corpus de Prasat Khna, elle n'avait pas été mentionnée par les différents chercheurs ayant inventorié ce site, à commencer par Étienne Aymonier (1901, p. 220-223), Étienne Lunet de Lajonquière (1907, p. 47-53) ou encore Henri Parmentier (1939, p. 194-211). Comme le soulignent Bruno Bruguier et Juliette Lacroix (2013, p. 139), ceci tient sans doute au fait qu'elle était masquée par des gravats, qui la protégeaient également des intempéries, ce qui explique qu'elle soit remarquablement bien conservée. En effet, 
Lunet de Lajonquière (1907, p. 51) souligne le mauvais état de conservation de la tour $\mathrm{E}$ et Parmentier (1939, p.199) précise: "La tour $E[. .$.$] est une tour de briques à$ fausses-portes avec baies à l'Est enterrée par les décombres. L'intérieur est inaccessible sans dégagement de la porte ». On ignore quand et dans quelles circonstances ce bâtiment a été dégagé, mais si la tour est toujours entourée de gravats, l'intérieur est en revanche à présent accessible (fig. 4).

Fig. 4. Inscription K. 1312 en cours d'estampage (Prasat Khna, piédroit nord de la tour E).

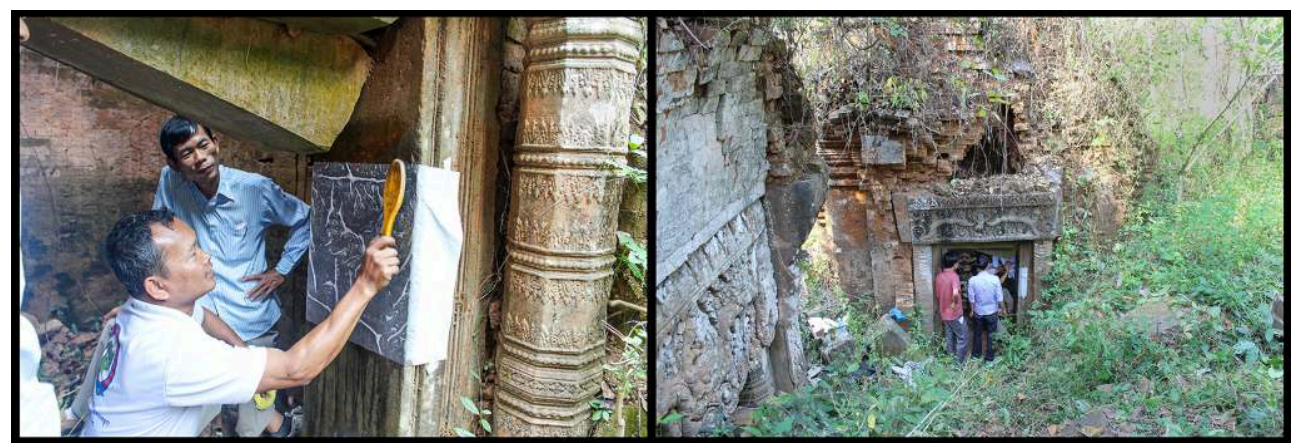

Cliché : Phoeung Dara.

Cette épigraphe a été publiée par Claude Jacques (2014, p. 402-405), János Jelen lui en ayant communiqué quelques photographies. L'interprétation qu'il en propose est globalement correcte, mais quelques fautes dans l'édition méritaient d'être corrigées. Nous en présenterons donc une édition et une traduction révisées avant de reprendre en détail quelques points, notamment d'ordre paléographique.

Le texte est composé de quatorze lignes en khmer angkorien. Il commémore une donation faite par un dignitaire, le Mrateñ Nirantarācārya, en faveur de deux des divinités qu'accueillait le Prasat Khna, les vrah kamrateñ 'añ Ta Rājya et Ta Sak Brāhma[ṇa], que nous avons déjà évoquées. Elle précise également le patrimoine foncier qui fait l'objet de cette donation et les transactions relatives à son acquisition.

Le nom du donateur, Nirantarācārya, n'est pas attesté par ailleurs dans les inscriptions, mais il est précisé qu'il était chef (khloñ) du temple (vnam) du kammrateñ jagat lingapura (1. 2). Ce nom est associé à plusieurs sanctuaires du Cambodge ancien. On le rencontre notamment à Koh Ker et il nous semble vraisemblable que c'est à ce site qu'il est fait ici allusion, sachant sa proximité avec celui de Prasat Khna et le lien particulier qui les unit par la présence du Kamraten் 'Añ Ta Rājya ${ }^{15}$. Au sujet de Lingapura, Cœdès (IC I, p. 69, n. 1) précisait : « le nom de Lingapura, qui a été porté par plusieurs localités, n'est pas susceptible d'une identification précise. Il a pu désigner tout ou partie du groupe de Koh Ker ». Si, dans l'inscription K. 1312, vnam kammraten jagat lingapura désigne le Prasat Thom, comme le pensait Claude Jacques (2014, p. 402), il faut donc traduire par : "le temple du Kamraten Jagat de Lingapura », ce toponyme devant être associé à la divinité principale de Koh Ker installée au Prasat Thom, c'est-à-dire Śiva Tribhuvaneśvara, qui est aussi désigné comme le Vrah Kamiaten 'Añ Jagat Ta Rājya dans les inscriptions ${ }^{16}$. 


\section{Texte ${ }^{17}$}

12 (1) ه $891^{18}$ śaka chatthī roc śrāvana sanaścaravāra ${ }^{19}$ nu mrateñ.ṇi(2)rantarācāryya ${ }^{20}$ khloñ· vnam் kammmraten' jagat• lingapura jau bhūmi (3) vrai travan்· khsac· ṇā sańk(u)ti ${ }^{21}$

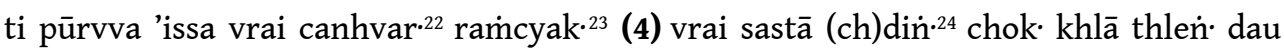
lvah ta gi vrai cassa nuva chdin. ${ }^{25}$ hara (5) thlen. dau lvah ta gi vrai cassa nuva gnań. dhār. ti pūrvva prasap. chdin'. ${ }^{26}$ lnya(6)n' ti jau nu bhājana ta vrah kammmraten' 'aña ta rājya nu vrah (7) kammmraten' 'aña ta sak vrāmhma nana ${ }^{27}$ bhājana jyaña $40^{28}$ nu thve ${ }^{29}$ pūjā vrah (8) ka[m]mraten்' 'añ' suvarṇ(ṇ)a $a^{30}$ • 'oy yajña ta vra[h] ka[m]mraten' 'añ' ta rājya lih (9) II ta vra[h] ka[m] mraten' 'añ' sak· vrāhma ${ }^{31}$ lih II pratidina • pādamūla ta (10) phjauva $^{32}$ bhūmī sastā chok khlā dhār pi jau • vāp vrau vāp vadeva ${ }^{33}$ vāp hari (11) devaparicāra ${ }^{34}$ vāp. 'āditya grāmapāla vāp. mādhava ${ }^{35}$ grāmapāla vāp.

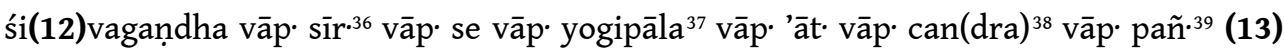
vāp. 'amṛta khloña vnvak' vāp. vurūna ${ }^{40}$ vāp. vrahma tammra(d)un'. ${ }^{41}$ kamilūna vāp. na(gi)(14)ya cām phjuh vāp' 'ap· cām phjụ

Fig. 5. Cliché de l'estampage de l'inscription K. 1312.

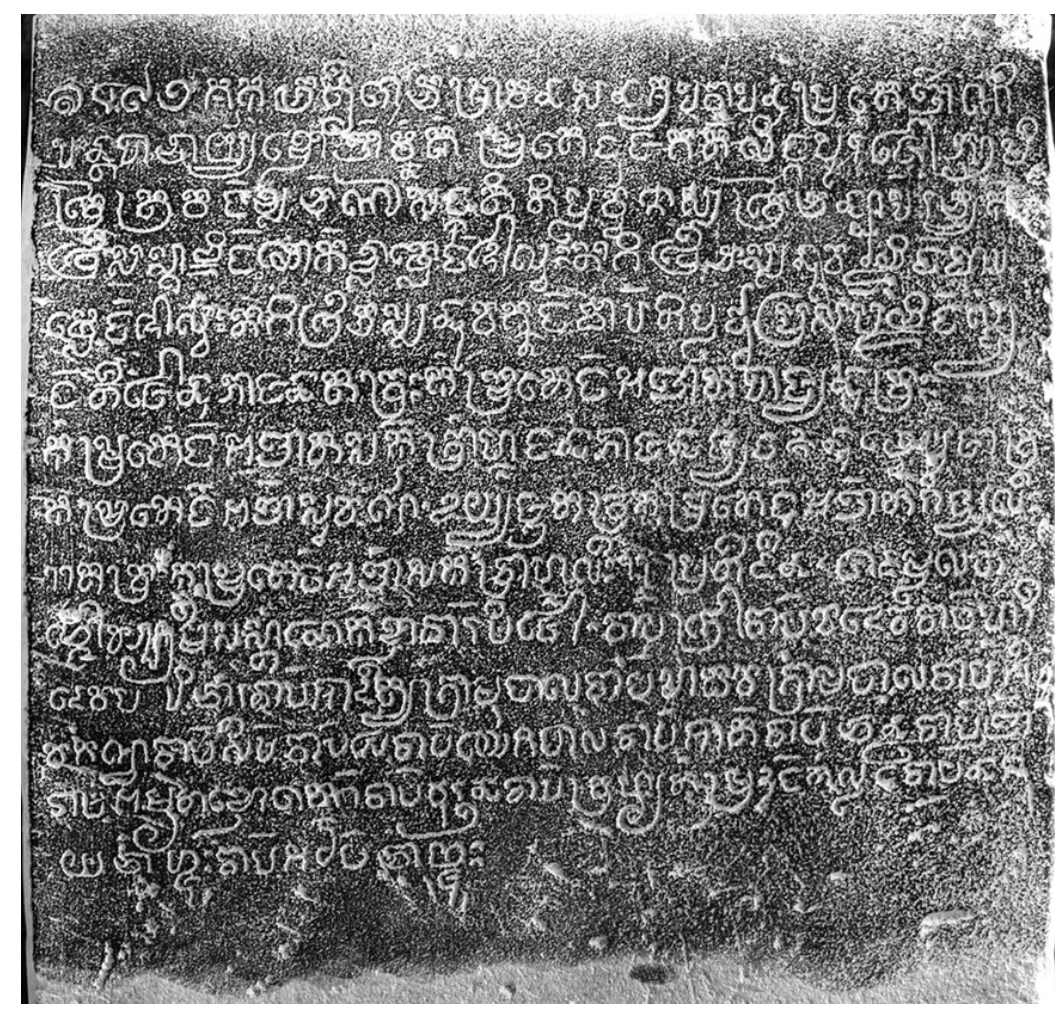

Cliché : Phoeung Dara. 
Fig. 6. Dates des inscriptions K. 1312 et K. 753.

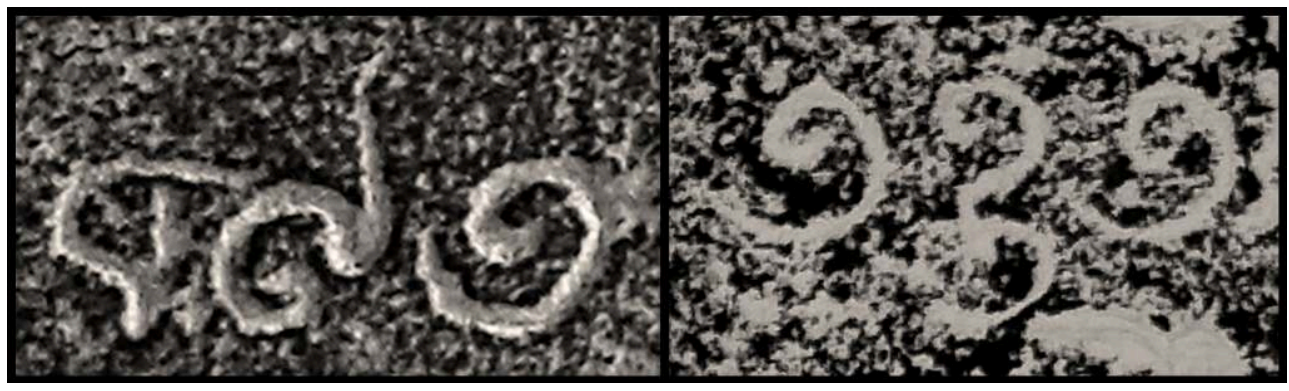

Cliché : Phoeung Dara ; estampage EFEO n. 950.2.

Fig. 7. Phjau (K. 1312, I. 10).

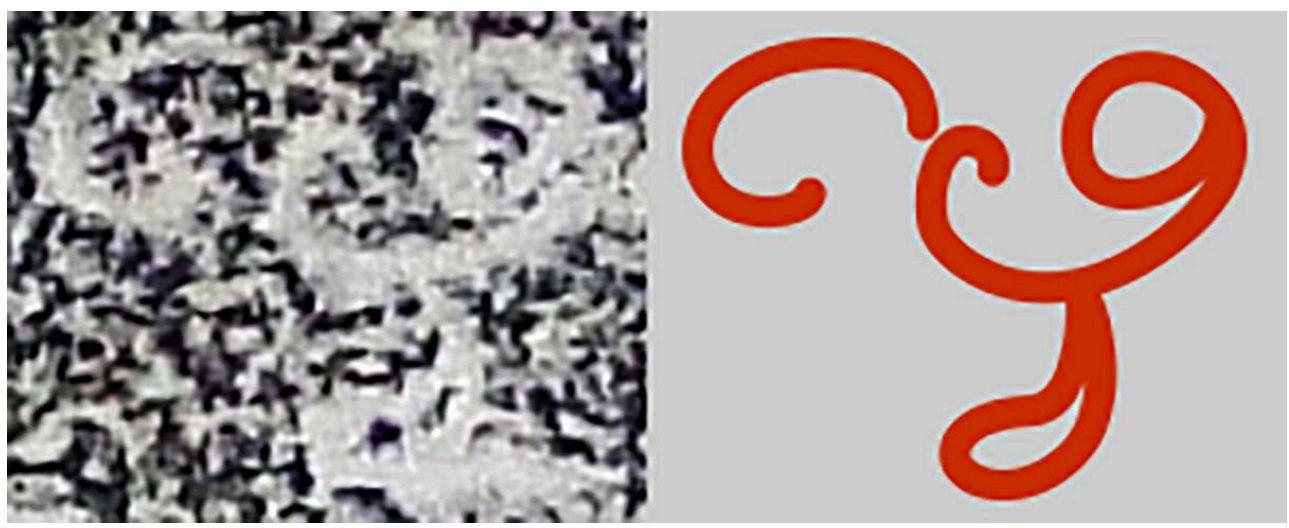

Cliché : Phoeung Dara, vectorisation : Dominique Soutif.

\section{Traduction}

13 (1) 891 śaka, sixième jour de la lune décroissante ${ }^{42}$ de (la mansion lunaire) śrāvana, un samedi.

14 (1-9) Alors, le Mrateñ Nirantarācārya, chef du temple du Kamiateñ Jagat (de) Lingapura, a acheté le domaine forestier (bhūmi vrai ${ }^{43}$ ) de Travang Khsac ( le bassin sableux ») à Sańkuti. À l'est, il se termine à Vrai Canhvar Ramcyak («la forêt du ruisseau des pandanus »). [Il comprend] ${ }^{44}$ la forêt Sastā, la rivière (chdiñ) de Chok Khlā («bosquet des tigres») en montant jusqu'à l'ancienne forêt (vrai cassa) et la rivière Hara, en montant jusqu'à l'ancienne forêt et le gnan் ${ }^{45}$ de Dhāra, bordé à l'est par la rivière Lnyan. [Tout cela] a été acheté avec un plat ${ }^{46}$, pour le Vrah Kamraten 'Añ ta Rājya et le Vraḥ Kamrateñ 'Añ ta Sak Brāhma ; le plat pesait 40 jyañ et il a fait la pūjā du Vraḥ Kamraten 'Añ Suvarṇa. Il donne en sacrifice chaque jour 2 lih ${ }^{47}$ au Vrah Kamraten 'Añ ta Rājya et 2 liḥ, au Vraḥ Kamrateñ 'Añ Sak Brāhma.

15 (9-14) Le vénérable ${ }^{48}$ a fait acheter les terres de Sastā, Chok Khlā (et) Dhār. Pour les acquérir : Vāp Vrau, Vāp Vadeva, Vāp Harī, serviteurs du dieu (devaparicāra), Vāp 'Āditya, gardien de village (grāmapāla), Vāp Mādhava, gardien de village, Vāp Śivagaṇdha, Vāp Sīr, Vāp Se, Vāp Yogipāla, Vāp 'Āt, Vāp Canda, Vāp Pañ, Vāp 'Amṛta, chefs d'équipe (khloñ vnvak), Vāp Varuṇa, Vāp Vrahma, Kamrateñ à l'intérieur [du temple], Vāp Nagiya, cāmm phjuh ${ }^{49}$ (et) Vāp Kap, cāmm phjuḥ. 


\section{La date} celle de 861 śaka dans son édition. Il faut effectivement lire un 9, bien que de forme un peu originale. Ceci est confirmé par Chris Eade qui, contacté à ce sujet, nous a précisé que le sixième jour de la lune décroissante de la mansion lunaire śrāvana coïncide bien avec un samedi en 891 śaka, mais pas en 861. Cette date correspond au samedi 7 août 969 de notre ère ${ }^{50}$. interrogation. En effet, il s'agit bien d'une spirale dextrogyre, sens de rotation habituellement utilisé pour le chiffre $1^{51}$, mais ce qui est plus inhabituel, c'est que la spirale s'enroule pour passer en dessous du chiffre et se termine en remontant vers la gauche. Ce caractère évoque plutôt un 6 , chiffre qui est presque toujours représenté par une spirale sénestrogyre, mais dont un exemple dextrogyre très similaire à notre chiffre des unités est connu dans l'inscription K. 753, qui date de 626 śaka $a^{52}$. Dans ce cas particulier, la lecture de deux six s'impose à moins d'envisager que cette épigraphe date de 121 śaka, ce qui est tout à fait improbable. Il est donc heureux que, dans le cas de K. 1312, la concordance établie par Chris Eade confirme également notre lecture du chiffre des unités.

Notons que la date de l'inscription, à ce jour la plus ancienne du site, invitait Claude Jacques (2014, p. 402) à supposer que le bâtiment 'E' était, de ce fait, le plus ancien de Prasat Khna. Cependant, les fouilles réalisées en 2020 dans le cadre du programme de recherche YAŚODHARĀŚRAMA témoignent de plusieurs phases de construction ${ }^{53}$ et, si notre hypothèse proposant de reconnaître le bâtiment ' $\mathrm{N}$ ' de Prasat Khna comme la chapelle d'un Yaśodharāśrama se confirme, celui-ci daterait au plus tard du début du $\mathrm{X}^{\mathrm{e}}$ siècle de notre ère. Il serait donc antérieur au bâtiment ' $\mathrm{E}$ ', ce qui laisse supposer la présence de bâtiments encore plus anciens car, rappelons-le, en province, les Yaśodharāśrama ont été installés à proximité de sanctuaires préexistants, des lieux saints, matérialisant ainsi la mainmise que le souverain exerçait sur l'ensemble de son territoire ${ }^{54}$. À ce sujet, il faut rappeler que Parmentier signalait que le Prasat Khna «possédait une annexe d'art khmèr primitif. Sur un point symétrique à l'édifice $D$ du plan de M. de Lajonquière est la ruine presque informe d'un petit sanctuaire de briques, qui offre la porte en large dalle de cet art. ${ }^{55}$, mais cette identification n'a pas encore pu être vérifiée au cours des campagnes de prospection et de fouilles du programme de recherche. Il s'agit d'une donnée particulièrement intéressante dans le cas du Prasat Khna, s'agissant de la région d'origine d'Hiranyadāma, le fondateur du culte du Kamraten 'Añ ta Rājya, étant donné l'importance que cette divinité prit dans le panthéon angkorien et nous nous attacherons donc à la vérifier et à dater cette structure avec plus de précision.

\section{L'objet du don}

19 Le détail de la transaction est plus complexe à saisir qu'il n'y paraît. Claude Jacques (2014, p. 404 ; cf. infra) propose l'interprétation suivante des lignes 3 à 7 : « la terre de Vrai Travang Khsac, vers le secteur oriental finissant à Vrai Canhur Cyak (et) Vrai Sastā, (au) stu'n Chok Khlā qui monte jusqu'à Vrai Cas, et le stu'n Hara, en montant

Bulletin archéologique des Écoles françaises à l'étranger , Asie du Sud-Est continentale 
jusqu'à Vrai Cas et Gnan Dhāra à l'est jusqu'au stu'n Lnyan் ». Cette traduction découle de son interprétation de sankkati (cf. n. 21), que nous déchiffrons plutôt sañkuti et considérons, faute de mieux, comme un toponyme. Bien que très inhabituelle et supposant un découpage du territoire inconnu par ailleurs, son interprétation de sankati ti pūrvva, "le secteur oriental» reste possible, mais in fine, ne change pas grand-chose à l'interprétation et n'éclaire pas la nature exacte du don. Il est vrai que la syntaxe est limitée et qu'il n'est pas évident de distinguer les éléments qui font l'objet de la donation des repères qui en fixent les limites, d'autant que cette façon de délimiter une terre ne fournit que des indices de bornages insuffisants et en tout cas imprécis en regard des terres bornées au quatre points cardinaux, voir aux directions intermédiaires, qui sont régulièrement mentionnées dans l'épigraphie ${ }^{56}$.

Notre proposition de restitution consiste à considérer que le domaine forestier de Travang Khsac ([comprend] la forêt Sastā [...]) est liée à la seconde énumération de la ligne 10 mentionnant l'achat de Sastā, Chok Khlā et Dhār ${ }^{57}$, qui invite à considérer les différentes composantes de la première comme des parties indépendantes d'une même donation, soit le domaine forestier de Travang Khsac qui n'est justement pas cité dans la seconde énumération; il faut d'ailleurs noter que l'interprétation proposée par Cœdès de bhümi vrai convient parfaitement si notre hypothèse s'avère exacte. Il reste étonnant que la rivière Hara soit également omise dans la seconde liste, mais nous ne voyons pas pour l'instant d'autre interprétation pour ce passage. Malheureusement, les terres en question n'ont pas pu être identifiées de nos jours.

Il demeure la question des protagonistes. Notre interprétation est liée à l'utilisation du causatif phjauv dans une phrase à la structure bien inhabituelle. Elle requiert de considérer la liste de personnes des lignes 9 à 14 comme les personnes impliquées dans la transaction, qu'elles aient été chargées de procéder à celle-ci, ou d'en être les témoins, à moins qu'il ne s'agisse plus simplement des vendeurs ; rien dans l'inscription ne précise leur rôle.

\section{Le Vraḥ Kaṁmraten் 'Añ ta Sak Vrāhma et le prix des terres}

À propos de la lecture vrāmhnanana qu'il propose à la ligne 7 de son édition, Claude Jacques (2014, p. 404, n. 194) note : «ce ne peut être ici que le célèbre Sak Brāhmaṇa, mais écrit de façon ahurissante", identification confirmée par l'occurrence vra[h] $k a[\dot{m}] m r a t e n \cdot$ ' 'añ' sak' vrāhma à la ligne 9. La présence d'un anusvāra avant le hma de la première mention de cette divinité est en effet un peu étrange, mais en dehors de cela, l'étonnement de Claude Jacques s'explique par une erreur de lecture - hma et non hnaet une erreur de découpage. Il faut en effet lire : vrāmihma nana. La syntaxe est bien un peu surprenante, car on attendrait le verbe nan, " peser » après l'objet - ou les objets auquel il fait référence (bhäjana), mais il nous semble évident qu'il se rapporte au poids qui est précisé, à savoir 40 jyañ.

Le nombre de plats n'est pas mentionné dans l'inscription, ce qui laisse supposer que c'est le poids de métal qui importait ici. Le poids en question permet pourtant de formuler une hypothèse. En effet, dans l'inscription K. 1218, par exemple, gravée sur un vase en bronze, est mentionné un poids de 3 tulà et 16 kâtikâ. La kātikā et le jyan étant tous deux équivalents à un vingtième de tulā, ce vase - de grande taille il est vrai - 
pesait donc 76 jyañ $^{58}$. Il est donc possible qu'il s'agisse d'un unique vase pesant 40 jyan். La valeur de ces mesures reste très incertaine et a pu évoluer dans l'espace et le temps, mais si la mesure mentionnée dans l'inscription K. 1218 correspond bien au poids du vase - environ $9 \mathrm{~kg}$ avec son support -, alors la quantité (de métal ?) de la transaction rapportée dans l'inscription K. 1312 était de 4,7 kg environ, ce qui est assez conséquent, quel que soit le métal utilisé.

Le fait que le matériau en question ne soit pas précisé est d'ailleurs intéressant. Bien que le "système monétaire" du Cambodge ancien se distingue par l'absence de monnaie proprement dite, il permettait des échanges relativement complexes, à commencer par l'achat de biens variés, mais aussi la pratique de prêts à intérêts, par exemple ${ }^{59}$. Afin de gérer au mieux ces transactions, on peut supposer l'existence d'un matériau « étalon " permettant d'établir une équivalence de valeur entre les différents biens. Un lecteur au fait de ce système n'aurait donc nul besoin que le matériau soit précisé, ce qui expliquerait cette lacune.

Dans le cas des mesures de capacité, on considère en général que le riz constituait l'étalon le plus naturel ; ici par exemple, le sacrifice quotidien offert aux dieux était donc vraisemblablement de deux lih [de riz] (l. 8-9). On peut supposer que, pour un vase, il s'agissait d'un métal précieux qui reste à préciser.

\section{Phle/thve}

Aux lignes 7-8, Claude Jacques propose de lire: [...] nu (phl)e pūjō vrah kamrateñ añ $s(u) v a r n ̣ n a$ et traduit : " [...] et le bénéfice de la pūjā de Monseigneur en or »; à propos du kamraten añ suvarna , il note également (2014, p. 404, n. 199) : « Il doit s'agir d'un linga recouvert d'or, mais où se trouve-t-il ? D'autre part, je comprends mal ce dernier membre de phrase ». De fait, ce passage peut paraître un peu obscur, mais il faut en premier lieu en corriger la lecture. Nous avons déjà évoqué la confusion de Claude Jacques entre pa et pha (cf. supra, n. 31). Sa lecture thve est l'occasion d'évoquer à présent sa confusion entre pha et tha. Ces deux caractères sont en effet assez proches, mais le fait que l'haste gauche de la consonne se courbe vers la droite permet de reconnaître un tha avec certitude. En outre, la lecture va-et non la-nous paraît évidente pour la souscrite (fig. 8). Une pūjā est donc faite (thve) à l'attention du Vrah Kamrateñ 'Añ Suvarṇa, sans doute afin de sceller la transaction. 
Fig. 8. Thve (K. 1312, I. 7).

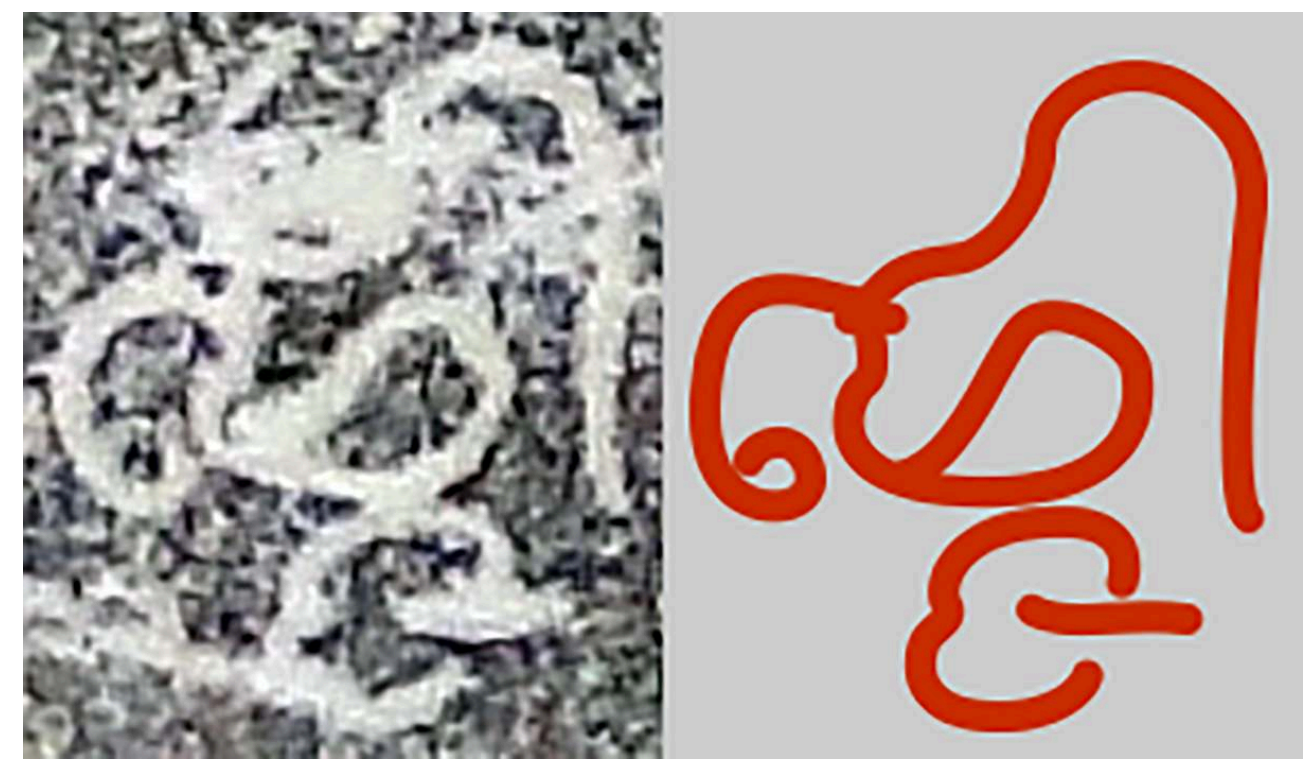

Cliché : Phoeung Dara ; vectorisation : Dominique Soutif.

L'identification de cette divinité n'en reste pas moins problématique. Même si théoriquement rien ne s'oppose à ce qu'il s'agisse d'un autre dieu que Śiva, l'hypothèse de Claude Jacques est envisageable, des linga de Śiva en or étant régulièrement cités dans le corpus épigraphique khmer, et ce dès la période préangkorienne. L'une de ces occurrences est particulièrement intéressante : un Kamraten Jagat Suvarṇalinga est mentionné comme bénéficiaire d'une donation dans l'inscription K. 136 qui provient de la région de Kratiéb ${ }^{60}$ aux côtés d'autres divinités parmi les plus célèbres du Cambodge ancien, au nombre desquelles on compte justement les Kamraten Jagat Lingapura (face c, 3) et Sak Vrāhmaṇa (face c, 1.9) mentionnées dans notre inscription; il s'agit donc bien, dans cette occurrence, de trois divinités distinctes. Cependant, le lieu de résidence de ce Suvarṇalinga n'a pas été identifié. Par ailleurs, même s'il est tentant de penser que le Suvarṇa de K. 1312 n'est autre que le Suvarṇalinga de K.136, nous ne pouvons l'affirmer.

Il est vraisemblable que cette pūjā ait été réalisée pour l'une des divinités du sanctuaire de Prasat Khna, suffisamment célèbre pour qu'il ne soit pas nécessaire de donner plus de détails à son sujet. Il nous paraît pourtant impossible que celle-ci soit le Śiva/ Kamraten் 'Añ Ta Rājya. En effet, il serait alors étrange qu'elle soit désignée comme le Kamraten 'Añ Ta Rājya juste après, à propos des prestations quotidiennes offertes aux deux divinités. Pourrait-il alors s'agir d'une autre divinité accueillie par ce temple, comme le Brahmā qui complète la triade, mais qui est, comme on l'a dit, désigné habituellement comme le kamraten் 'añ ta 'cas, «l'ancien »? La question reste ouverte, d'autant que l'on ignore la divinité qu'abritait le sanctuaire E, sur la porte duquel l'inscription a été gravée.

Le fait que la donation soit le fait du chef du temple de Lingapura, dans lequel nous reconnaissons, comme Claude Jacques, le sanctuaire de la divinité principale de Koh Ker, le Vraḥ Kamraten் 'Añ Jagat Ta Rājya que l'on désigne le plus souvent comme le Devarāja, constitue en tout cas un nouveau témoignage du lien particulier qu'entretenait le sanctuaire de Prasat Khna avec cette dernière. Il est en effet assez naturel que le chef du sanctuaire de Koh Ker fasse une donation au temple qui abritait 
le Kamraten் 'Añ Ta Rājya et une divinité rendant hommage à son fondateur, le «brāhmane scythe » Hiranyadāma.

C'est sans doute à la présence de ces divinités de premier plan que l'on doit le prestige et, en conséquence, la monumentalité de ce sanctuaire dans un site pourtant très éloigné de la capitale, ainsi que l'installation successive de fondations royales importantes dans son enceinte, ou à proximité, à commencer par celles d'āśrama sous les règnes de Yaśovarman $\mathrm{I}^{\mathrm{er}}$ et de Sūryavarman $\mathrm{I}^{\mathrm{er}}$ et d'une chapelle d'hôpital par Jayavarman VII, qui suggère la permanence d'un établissement humain jusqu'à la fin du XII $s$.

\section{BIBLIOGRAPHIE}

\section{Abréviations}

APK : Articles sur le pays khmer ; cf. CœDÈs 1989-1992.

BEFEO : Bulletin de l'École française d'Extrême-Orient.

CEFEO : Cahiers de l'École française d'Extrême-Orient.

IC : Inscriptions du Cambodge ; cf. CÆDÈs 1937-1966.

\section{Bibliographie}

AYMONIER 1901

Étienne Aymonier, Le Cambodge, tome II, Paris, Ernest Leroux, 1901.

BOURDONNEAU 2011

Éric Bourdonneau, « La fondation du culte du Devarāja. Danse, sacrifice et royauté au Prasat Thom de Koh Ker ", Comptes rendus des séances de l'Académie des Inscriptions et Belles-Lettres, $155^{\mathrm{e}}$ année, n. 3, 2011, p. 1343-1382.

BOURDONNEAU 2016

Éric Bourdonneau, « La stèle de Sdok Kak Thom et le Devarāja. Récits et acteurs d'une 'naissance' ", in Nasir Abdoul-Carime, Grégory Mikaelian, Joseph Thach (éd.), Le passé des khmers. Langues, textes, rites, Berne, etc., Peter Lang, 2016, p. 115-165.

BRUGUIER, LACROIX 2013

Bruno Bruguier, Juliette Lacroix, Preah Khan, Koh Ker et Preah Vihear : les provinces septentrionales, Phnom Penh, Japan Printing House, 2013.

CHEA SOCHEAT, ESTÈVE, SOUTIF, SWENSON à paraître

Chea Socheat, Julia Estève, Dominique Soutif, Edward Swenson, « Aśramas, Shrines and Royal Power », in Damian Evans, Miriam Stark, Mitch Hendrickson (éd.), The Angkorian World, Routledge Worlds series, Londres, New York, Routledge, à paraître. 
CCEDÈs 1911

George Cœdès, «Études Cambodgiennes : 5. Une inscription d'Udayādityavarman I, 6. Des édicules appelés "bibliothèques" ", BEFEO 11 (3-4), 1911, p. 400-404, 405-406 [Réimpr. 1989, APK 1, p. 10-15, 15-16].

CEDÈS 1937-1966

George Cœdès, Inscriptions du Cambodge (8 vol.), Hanoi, Paris, EFEO, 1937-1966.

CEDÈs 1943

George Cœdès, « Études Cambodgiennes : 37. Le site de Janapada, d'après une inscription de Pràsàt Khna », BEFEO 43, 1943, p. 8-11.

CEDÈS 1989-1992

George Cœdès, Articles sur le pays khmer (2 vol.), Paris, EFEO, 1989-1992 [Réimpr. des articles sur le Cambodge parus dans le BEFEO et dans les CEFEO].

CEDÈs, DUPONT 1943

George Cœdès, Pierre Dupont, «Les stèles de Sdŏk Kăk Thom, Phnom Sandak et Práh Vihār », BEFEO 43, 1943, p. 56-134 [Réimpr. 1992, APK 2, p. 56-154].

ESTÈVE, SOUTIF 2010-2011

Julia Estève, Dominique Soutif, « Les Yaśodharāśrama, marqueurs d'empire et bornes sacrées : conformité et spécificité des stèles digraphiques khmères de la région de Vat Phu », BEFEO 97-98, 2010, p. 331-355.

GRIFFITHS, SOUTIF 2008-2009

Arlo Griffiths, Dominique Soutif, « Autour des terres de Loñ Śrī Vișṇu et de sa famille : Un document administratif du Cambodge angkorien, l'inscription K. 1238 », BEFEO 95-96, 2008-2009, p. 29-72.

HEADLEY, CHIN, OK 1997

Robert K. Jr. Headley, Rath Chin, Soeum Ok, Modern Cambodian English Dictionary, Kensington, Dunwoody Press, 1997.

JACQUES 1971

Claude Jacques, « Supplément au Tome VIII des Inscriptions du Cambodge », BEFEO 58, 1971, p. 177-195.

JACQUES 2014

Claude Jacques, Koh Ker : Temples et inscriptions avec une étude sur le roi Jayavarman IV et un essai sur l'« esclavage » dans l'ancien pays khmer, s.l., Hungarian Southeast Asian Research Institute, 2014.

JENNER 2009

Philip N. Jenner, A Dictionary of Angkorian Khmer, Canberra, Pacific Linguistics, 2009.

LUNET DE LAJONQUIÈRE 1907

Étienne Edmond Lunet de Lajonquière, Inventaire descriptif des monuments du Cambodge, tome II, Paris, Ernest Leroux, 1907.

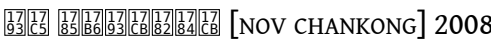

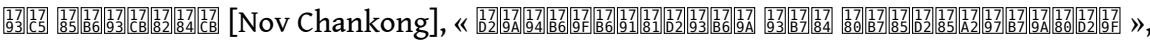

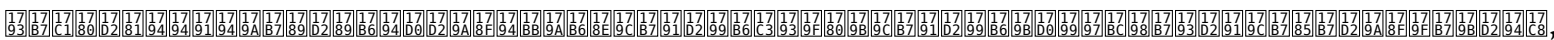

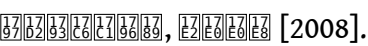

PARMENTIER 1927

Henri Parmentier, L'Art khmèr primitif (2 vol.), Paris, EFEO, 1927. 
PARMENTIER 1939

Henri Parmentier, L'Art Khmer Classique. Monuments du quadrant Nord-Est (2 vol.), Paris, EFEO, 1939.

POU 2004

Saveros Pou, Dictionnaire vieux khmer-français-anglais. An Old Khmer-French-English Dictionary. Vacanānukram khmaer cas'-paramn-angles, Paris, L'Harmattan, 2e édition, 2004 [1 ${ }^{\text {re }}$ éd. $1992+$ supplément].

SOUTIF 2008

Dominique Soutif, « Dénombrer les biens du dieu ; étude de la numération du khmer ancien $\left(\mathrm{VI}^{\mathrm{e}}-\mathrm{XII}{ }^{\mathrm{e}}\right.$ siècle śaka)», Siksâcakr 8, 2008, p. 51-80 [paru en 2010 ; traduction khmère, p. 172-206]. SOUTIF 2009

Dominique Soutif, « Organisation religieuse et profane du temple khmer du VII au XIII $^{\mathrm{e}}$ siècle », thèse de doctorat sous la direction de Michel Jacq-Hergoualc'h, Université de Paris III Sorbonne nouvelle, 2009, 3 vols.

SOUTIF 2018

Dominique Soutif, « The Corpus of Khmer Inscriptions: State of the Art, Methods and First Results », in Daniel Perret (éd.), Writing for Eternity: A Survey of Epigraphy in Southeast Asia, Paris, EFEO, 2018, p. 151-161.

SOUTIF et al. 2019, 2020

Dominique Soutif, Julia Estève, Chea Socheat, Edward Swenson, Rapports annuels de la mission Yaśodharāśrama, rapports non publiés, Siem Reap, MOCFA-APSARA-MAE-EFEO, 2019, 2020.

\section{NOTES}

1. NOV CHANKONG 2008, p. 27.

2. Ce temple a été inventorié par Lunet de Lajonquière sous le numéro 315 (LUNET DE LAJONQUIÈRE 1907, p. 47-53) et par le CISARK sous le numéro 627 (Carte Interactive des Sites Archéologiques Khmers). On se reportera à ces travaux ainsi qu'à ceux de PARMENTIER 1939 (p. 194-211) pour une description détaillée des bâtiments de ce site.

3. La troisième enceinte, qui est composée d'une simple levée de terre alors que les deux autres sont constituées d'un mur en latérite, a été identifiée grâce aux levés topographiques réalisés par le programme de recherche archéologique YAŚODHARĀŚRAMA qui est financé par la commission des fouilles du Ministère de l'Europe et des Affaires étrangères, et s'inscrit également dans le projet DHARMA (SOUTIF, ESTÈVE, CHEA SOCHEAT, SWENSON 2019). Les bâtiments du temple ont été désignés par des lettres par Lunet de Lajonquière, système repris pour le plan dressé par Parmentier (PARMENTIER 1939, pl. XXVII), complété par Bruguier et Lacroix (BRUGUIER, LACROIX 2013, p. 135, plan 19) et par notre programme de recherche.

4. CHEA, ESTÈVE, SOUTIF, SWENSON, à paraître.

5. La forme de la stèle qu'il abritait, qui porte l'inscription K. 1430, est conforme à celle des inscriptions digraphiques commémorant la fondation des āśrama de Yaśovarman I ${ }^{\mathrm{er}}$ en province. Son texte originel a apparemment été volontairement effacé et elle a été réinscrite, sans doute aux environs $\mathrm{du} \mathrm{xI}^{\mathrm{e}}$ siècle de notre ère. Il s'agit pour l'instant 
d'une estimation paléographique, le texte, difficilement déchiffrable, étant encore inédit.

6. SOUTIF et al. 2020.

7. LUNET DE LAJONQUIÈRE 1907, n 316, p. 53.

8. SOUTIF et al. 2019, 2020.

9. K. 355 (IXe s. śaka ; CÆDÈs 1911, p. 405-406), K. 356 (902 śaka ; CÆEDÈs 1911, p. 400-404 \& 1943, p. 10), K. 660 (963 śaka ; IC I, p. 195-197), K. 661 (xe s. śaka ; IC I, p. 197-219), K. 1312 (891 śaka ; JACQUES 2014, p. 402-405) et K. 1340 (angkorienne; inédite). À cela, il faut ajouter une inscription incomplète et quelques fragments découverts -comme K. 1340 - au cours des travaux du programme de recherche YAŚODHARĀśRAMA (SOUTIF et al. 2019). Ces fragments n'ont pas encore été inventoriés par le CIK.

10. CEDÈs 1911, p. 405-406. À ce sujet, cf. SOUTIF 2009, p. 331-333.

11. K. 356 N, l. 5 ; 902 śaka ; CCDÈs 1943, p. 10-11.

12. K. 235 C, 1. 71-74 ; 974 śaka ; CCEDÈs, DUPONT 1943, p. 87, 108-110.

13. BOURDONNEAU 2016, p. 128-129.

14. JACQUES 2014, p. 403.

15. Cf. BOURDONNEAU 2011

16. BOURDONNEAU 2011, p. 1345-1346 et 1352-1361.

17. Cette édition est fondée sur des clichés et estampages réalisés en janvier 2013, à l'occasion d'une prospection organisée par les auteurs et Ang Choulean, en collaboration avec l'équipe d'épigraphie de l'Autorité pour la Protection du Site et l'Aménagement de la Région d'Angkor (APSARA). Nous tenons à remercier ici toutes les personnes ayant participé à cette visite de site (fig. 5).

18. Claude Jacques : 861 (cf. infra).

19. Sanaścaravāra : sanaiścaravāra śanaiścaravāra (JENNER 2009, s.v.).

20. La distinction $i / \bar{i}$ n'est pas faite dans cette inscription. La forme en spirale inciterait à lire $\bar{i}$, mais cette lecture occasionnerait de nombreuses fautes par ailleurs et nous restituerons donc ici systématiquement l'orthographe correcte. En outre, il faut corriger nirantara $a^{\circ}$ en nirantara ${ }^{\circ}$. On notera que la cacuminale est également utilisée à la place de la dentale à la ligne suivante où il faut corriger ṇā en $n \bar{a}$.

21. JACQUES 2014: sañkati. Sańkuti comme sankkati sont des hapax. Claude Jacques proposait de voir dans sańkati une variante de sankkat, " part, côté, section » (POU 2004, s.v.) qui explique l'interprétation qu'il propose de ce passage (cf. infra, n. 46) ; cela dit, ce mot n'apparaît qu'une fois dans le corpus angkorien, dans un contexte très différent (K. 470, 1.10; 1249 śaka; IC II, p.187-188). Notons qu'il est possible que le ti ait été redoublé et/ou que la voyelle $i$ ait été notée par erreur; il faudrait donc envisager les lectures sańkati ti, sańku ti ti, sañkut ti ou encore sańkat ti.

22. JACQUES 2014 : canhur cyak; il suppose qu'il s'agit d'une forme inédite de canhvar, «canal» (2014, p. 404, n. 191), mais la lecture canhvar nous semble plus correcte en dépit de la petite taille de la boucle du va souscrit. On notera que le $r a$ de canhvar comporte deux hastes - usage plutôt préangkorien -, alors que celui de ramcyak n'en a qu'une.

23. JACQUES 2014 : cyak. 
24. On est d'abord tenté de lire cdin, comme Claude Jacques, mais outre le fait qu'il s'agisse plutôt de la forme préangkorienne (JENNER 2009, s.v.), l'akșara ca serait alors très différent des autres occurrences de cette inscription. Il faut cependant reconnaître que ce cha est également dissemblable de celui du mot chok qui le suit immédiatement. Des trois occurrences de chdin, seule la seconde, à la fin de la ligne 4, comporte un cha parfaitement tracé. Les deux autres suivent un autre ductus qui pourrait inciter à lire

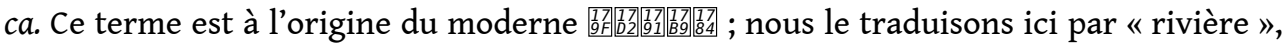
mais il peut s'agir de cours d'eau relativement modestes (HEADLEY et al. 1997, s.v.: «small river, stream »).

25. JACQUES 2014 : chdin.

26. JACQUES 2014 : cdin.

27. JACQUES 2014 : vrāmihnanana (cf. infra).

28. JACQUES $2014: 20$ : la boucle supérieure de ce caractère est manifestement une barre additive imposant la lecture 40 (souTIF 2008, p. 60).

29. JACQUES 2014 : phle (cf. infra).

30. JACQUES 2014: s(u)varnnna. Le (na/na) souscrit est difficile à déchiffrer, mais la souscrite évoque ici un ṇa de taille réduite ressemblant à une petite sinusoïde que l'on rencontre par ailleurs, bien qu'assez rarement (cf., par exemple, K. 1198, face A, 1. 5 ; estampage EFEO n. 1654).

31. Jacques $2014: v \operatorname{va} h(m a)[n a]$ (cf. infra).

32. Ibid. : $p \bar{a}(10) \tilde{n} j a u v a$. La lecture pāñjauva proposée par Claude Jacques (1.9-10) nous semble erronée. Il note : « la forme du ñ ne se laisse deviner qu'à cause du ja souscrit. Ce mot semble être un hapax: il est clairement dérivé de jau, précédé du causatif $p a »$ (JACQUES 2014, n. 202, p. 404). Si la lecture du ja est certaine, les autres caractères nous semblent plus douteux. Le dernier akșara de la ligne 9 est en effet clairement un ta et le premier de la ligne 10 un pha: l'haste droite de ce caractère revient nettement vers l'arrière en faisant une boucle vers le bas (fig. 7). Il faut donc lire «ta phjauva ». On ne connaît qu'une autre occurrence de ce terme, mais avec une orthographe différente, phjov, dans l'inscription inédite K. 1186 (face A, 1.18 ; estampage EFEO n. 1684). Il s'agit en tout cas bien d'un dérivé causatif de jov $(\sim$ jau $\sim$ jauv $\sim$ jo $\sim$ jā), « acheter » (JENNER 2009, s.v.).

33. Comme le suppose Claude Jacques (2014, p. 404, n. 204), il nous semble probable que la première syllabe de cet anthroponyme a été omise (śivadeva, navadeva, śravadeva ?).

34. Ibid. : hari(11) deva paricā I. Cette lecture s'explique par le fait que, comme dans le cas de canhvar (1.3), un ra à double haste est utilisé pour l'akșara portant la voyelle $i$, alors que le second ne comporte qu'une haste et peut donc être confondu avec un danda.

35. Ibid. : hadhava. Ce mot n'est pas attesté dans le corpus et la lecture mādhava, terme connu entre autres comme anthroponyme, nous semble donc plus vraisemblable, d'autant qu'on distingue une barre horizontale entre les deux hastes. Il faut cependant noter que la voyelle $\bar{a}$ devrait rejoindre la première haste et non la seconde.

36. Ibid. : lìr. La position de la voyelle au-dessus de la boucle gauche et non de l'haste droite impose cette lecture.

37. Ibid. : yogapāla. 
38. Ibid. : candu. Bien que le da souscrit soit difficile à déchiffrer et que le ra souscrit ne remonte pas sur la gauche - faute de place, a priori -, la lecture candra nous semble plus vraisemblable.

39. Ibid. : vāp ña. Le second $p a$ - qui est souscrit - est discret et se confond avec le virāma du vāp de la fin de la ligne 13, mais il nous semble certain.

40. Claude Jacques propose de corriger en varuna, bien que, selon lui, ce nom ne soit pas attesté dans le corpus (2014, p. 405, n. 210). Nous en avons pourtant trouvé plusieurs occurrences en tant qu'anthroponymes (par exemple si varuna, K. 231, 1.6; 888 śaka; IC III, p. 72). Cette correction nous parait donc d'autant plus vraisemblable.

41. Claude Jacques (2014) lit plutôt kammra(du)nं, qu'il propose de corriger en kammraten. Le ka est douteux et ressemble à un ta, mais cette correction nous semble correcte, faute d'interprétation des hapax $(k / t) a \dot{m} m r a(d u) \dot{n}$ et $(k / t) a \dot{m m r a}(t u) \dot{n}$. On peut supposer que les Vāp Varuna et Vrahma occupaient une fonction "à l'intérieur (kamilūin) [de l'enceinte du temple]», comme dans les inscriptions K. 89 (khloñ vala kamilun kamven, «chef de la population à l'intérieur de l'enceinte »; 1.11;924 śaka ; IC III, p. 166-167) ou K. 134 ('nakk pamre kamilun vrah, «serviteur dans le temple»; 1.10 ; 703 śaka ; IC II, p. 93-94).

42. Et non «montante » comme le notait Claude Jacques (2014, p. 404).

43. Nous suivons ici l'interprétation de bhümi vrai proposée par Cœdès (IC VI, p. 178 ; cf. infra).

44. À propos de cette interprétation, cf. infra.

45. Philip N. Jenner conjecture le sens de "place to live, dwelling-place, residence " (2009, s.v.), mais dans l'attente d'un contexte plus précis, la traduction proposée par Saveros Pou, «barreau de clôture. Clôture» (2004, s.v.), qui renvoie au moderne

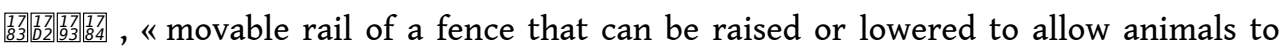
enter or exit » (Headley et al. 1997, s.v.) nous paraît plus satisfaisante ; il pourrait donc s'agir ici d'un enclos.

46. Ce singulier est conjectural (cf. infra).

47. De riz vraisemblablement (Jacques 2014, p. 404, n. 201 ; cf. infra).

48. Il n'est pas précisé qui est ce pādamūla. On peut se demander s'il s'agit du Mrateñ Nirantarācārya ou du vénérable du temple récipiendaire de la donation.

49. Cette fonction, qui apparaît également dans l'inscription K. 391 (0, 1. 21 ; 1004 śaka ; IC VI, p. 298, 299, n. 2), n'a pas encore été clairement élucidée. Comme le signalait George Cœdès, pjuh ( phjuh), et son dérivé pamjuh, s'appliquent régulièrement à des religieux (IC V, p. 84, n. 3). Philip N. Jenner conjecture le sens de pjuh « servir » et par extension celui de cāmm phjuh (2009, s.v.), « [personne qui] supervise le service », ce qui explique l'interprétation proposée par Claude Jacques, « gardien des services » (2014, p. 55).

50. Comm. pers., février 2013.

51. SOUTIF 2008, p. 55 et ill. 12, p. 75.

52.1. 1 ; IC V, p. 58 ; fig. 6.

53. sOUTIF et al. 2019, 2020.

54. ESTÈVE, SOUTIF 2010-2011.

55. PARMENTIER 1927, p. 170. 
56. Par exemple K. 206, 1. 6-14 ; 964 śaka ; IC III, p. 12-14.

57. Claude Jacques rassemble alors les deux derniers en un seul toponyme (2014, p. 404).

58. 929 śaka ; SOUTIF 2009, p. 146-147 et 593-598.

59. GRIFFITHS, SOUTIF 2008-2009, p. 54-55.

60. Face c, 1. 17 ; $\mathrm{x}^{\mathrm{e}}$ s. śaka; Lovek ; IC VI, p. 285-286.

INDEX

oeuvres https://ark.frantiq.fr/ark:/26678/pcrt3NZgrIT6pt

lieux https://ark.frantiq.fr/ark:/26678/pcrtJroNM0pDZ7

Thèmes : EFEO

Année de l'opération : 2020

sujets https://ark.frantiq.fr/ark:/26678/pcrtJrInD7k8G1, https://ark.frantiq.fr/ark:/26678/ pcrt30jRVudWjq, https://ark.frantiq.fr/ark:/26678/pcrtYhYMiLwDUr, https://ark.frantiq.fr/ ark:/26678/pcrt1hEGf8Mxjm, https://ark.frantiq.fr/ark:/26678/pcrt6PQaIIfrpT

\section{AUTEURS}

DOMINIQUE SOUTIF

École française d'Extrême-Orient

JULIA ESTÈVE

Université Mahidol, Bangkok 